\title{
Long-term follow-up of over 600 living-related kidney donors: single center experience
}

\author{
Burak Sayin ${ }^{1}$, Aydincan Akdur ${ }^{2}$, Emre Karakaya² ${ }^{2}$ Ebru H. Ayvazoglu Soy² ${ }^{2}$ Mehmet Haberal ${ }^{2}$ \\ ${ }^{1}$ Department of Nephrology, Baskent University, Ankara, Turkey \\ ${ }^{2}$ Department of Surgery, Baskent University, Ankara, Turkey
}

Background: The number of deceased donors is inadequate. Therefore, living kidney donations are increasing day by day. However, long term complications after donation are still unclear. We aimed to determine the long-term results of living-related kidney donors.

Methods: Since 1986 we performed 2,188 kidney transplant in our centers, and 1,788 of them were living-related kidney transplant (LRKT). Before donation all donor candidates were evaluated according to the Baskent University criteria. Out of 607 donors, 236 donors were evaluated in the outpatient clinic and the rest were evaluated by teleconference method. We performed obtaining detailed anamnesis clinical examination, blood tests and ultrasonogram evaluations to donors.

Results: We evaluated the long-term results of our 607 patients, of which 428 were female. The mean age of the donors was $52.03 \pm 11.54$ years. Mean time after donation was $10.4 \pm 3.2$ years. The estimated glomerular filtration rate was $77 \pm 16 \mathrm{~mL} / \mathrm{min}$ in our donor population. None of our donors developed end-stage renal disease. Twenty-four donors (3.9\%) are diagnosed with diabetes, 21 donors (3.4\%) with thyroid disease, 64 donors (10.5\%) with hypertension and 48 donors (8.8\%) with atherosclerotic cardiovascular disease. One hundred and seventy-four donors (28.6\%) developed mild to moderate obesity. Only five patients developed malignity and all of these were diagnosed at least 10 years after donation.

Conclusions: We showed that $28.6 \%$ of donors developed obesity that increased the risk of systemic disease and $26.6 \%$ of donors developed systemic disease in the long-term follow-up period. Also, the unrelated donors may be desperate if a family member needed donation in the future. Therefore, we recommend that deceased donor kidney transplant (DDKT) should be first choice. If DDKT is not possible, LRKT should be preferred. 